\title{
Inhibition of endothelial lipase causes increased HDL cholesterol levels in vivo
}

\author{
Weijun Jin, John S. Millar, Uli Broedl, Jane M. Glick, and Daniel J. Rader
}

Department of Medicine and Center for Experimental Therapeutics, University of Pennsylvania School of Medicine, Philadelphia, Pennsylvania, USA

\begin{abstract}
Endothelial lipase (EL) is a recently discovered member of the lipoprotein lipase gene family that hydrolyzes HDL phospholipids ex vivo and reduces HDL cholesterol (HDL-C) levels when overexpressed in vivo in mice. To gain further insight into the physiological role of EL in the metabolism of HDL in vivo, studies were performed in which EL was inhibited in wild-type, hepatic lipase knockout $\left(H L^{-/-}\right)$, and human apoA-I transgenic mice by intravenous infusion of a polyclonal antibody inhibitory to murine EL. As compared with infusion of a control antibody, infusion of the inhibitory antibody resulted in a $25-60 \%$ increase in HDL-C levels in the three mouse models, with the peak HDL-C levels occurring at 48 hours after injection. Inhibition of EL also generated larger HDL particles in the $\mathrm{HL}^{-/-}$ mice. The clearance of HDL phospholipid was significantly slower in human apoA-I transgenic mice injected with an antibody against murine $\mathrm{EL}(\mathrm{mEL})$ than in mice injected with a control antibody. We conclude that inhibition of EL results in increased HDL-C levels and that EL is an important enzyme in the physiological regulation of HDL metabolism.
\end{abstract}

J. Clin. Invest. 111:357-362 (2003). doi:10.1172/JCI200316146.

\section{Introduction}

Endothelial lipase (EL) is a recently discovered member of the triglyceride lipase gene family $(1,2)$. EL is highly homologous to lipoprotein lipase (LPL) and hepatic lipase (HL), both of which are known to hydrolyze lipids within lipoproteins and thereby influence their metabolism. EL has been shown to effectively hydrolyze HDL phospholipids in vitro (3), and overexpression of human EL in mouse liver markedly reduced plasma HDL cholesterol (HDL-C) levels in vivo (1). These data suggested that EL might play a physiological role in modulating HDL metabolism. However, proof of that concept requires an assessing the effect of reduction of EL activity in vivo.

Antibody inhibition has been used to gain insight into the physiological roles of enzymes in vivo. Indeed, the roles of LPL and HL in lipoprotein metabolism were established in part through antibody inhibition studies in rats, chickens, and monkeys (4-9). Because overexpression of EL markedly reduced HDL-C levels

Received for publication June 11, 2002, and accepted in revised form November 12, 2002.

Address correspondence to: Daniel J. Rader, University of Pennsylvania School of Medicine, 654 BRB II/III, 421 Curie Boulevard, Philadelphia, Pennsylvania 19104, USA.

Phone: (215) 898-4011; Fax: (215) 573-8606;

E-mail: rader@mail.med.upenn.edu.

Conflict of interest: The authors have declared that no conflict of interest exists.

Nonstandard abbreviations used: endothelial lipase (EL); HDL cholesterol (HDL-C); hepatic lipase (HL); murine EL (mEL); lipoprotein lipase (LPL); murine HL (mHL); murine LPL (mLPL); fast protein liquid chromatography (FPLC); $\left[{ }^{3} \mathrm{H}\right]$ methylcholinedipalmitoyl phosphatidylcholine $\left(\left[{ }^{3} \mathrm{H}\right]\right.$ methylcholine-DPPC). in mice, we hypothesized that antibody inhibition of EL would increase HDL-C levels. Here, we report the results of several independent experiments in which we used a specific polyclonal antibody against murine EL (mEL) to inhibit EL in mice. Our results demonstrate that inhibition of EL in mice results in a significant increase in HDL-C levels and, in the absence of HL, in HDL particle size.

\section{Methods}

Generation of a rabbit polyclonal antibody to murine EL. A recombinant adenoviral vector containing the $\mathrm{mEL}$ cDNA was made using methods described previously (1) and used to immunize a rabbit. Viral particles $\left(5 \times 10^{12}\right)$ were injected into a New Zealand white rabbit (HareMarland, Hewitt, New Jersey, USA) through the ear vein. Sera were obtained at various intervals for analysis of antibody reactivity to $\mathrm{mEL}$ by Western blotting. Control serum was generated using the injection of a recombinant adenovirus that contains no transgene. Three months after injection, rabbits were anesthetized and exsanguinated. Serum was separated from blood cells and stored in aliquots at $-80^{\circ} \mathrm{C}$. The $\operatorname{IgG}$ fractions were precipitated from the sera using ammonium sulfate (10) and purified using a protein $\mathrm{G}$ column (Amersham Pharmacia Biotech, Uppsala, Sweden) according to the manufacturer's protocol.

In vitro inhibition and immunoblotting of $m E L$. Transfection of expression plasmids containing $\mathrm{mEL}$, murine HL (mHL), and murine LPL (mLPL) cDNAs was performed in HEK293 cells using lipofectamine (Invitrogen, Carlsbad, California, USA). Conditioned media were collected 48 hours after transfection. Aliquots of 

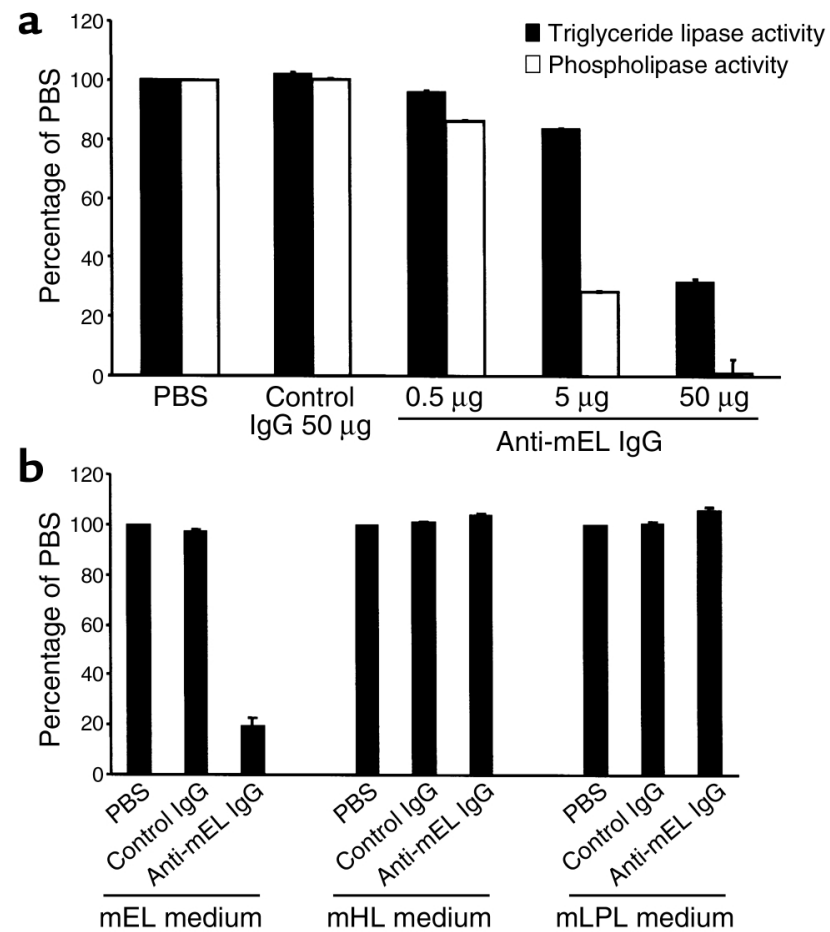

C

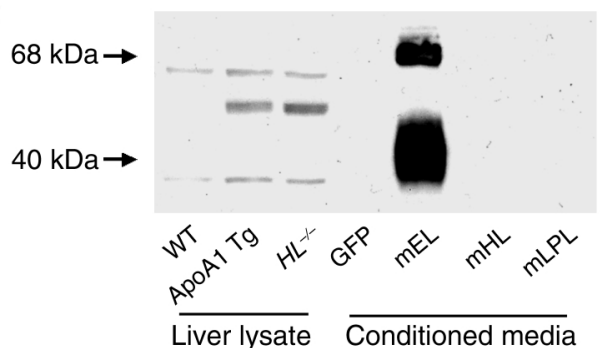

conditioned medium were incubated with an equal volume of PBS containing anti-mEL IgG or control IgG for 1 hour at $4{ }^{\circ} \mathrm{C}$. Triglyceride lipase and phospholipase activities were measured in triplicate as previously described (3). One unit of lipase activity is defined as liberating $1 \mathrm{nmol}$ of free fatty acid per hour.

Conditioned media from mEL-, mHL-, and mLPLtransfected cells $(10 \mu \mathrm{l})$ and homogenized liver lysates ( $15 \mu \mathrm{g}$ of protein) from wild-type, human apoA-I transgenic, and $H L^{-/-}$mice were immunoblotted with the anti-mEL antibody. Media and lysates were mixed with Laemmli sample buffer and heated at $85^{\circ} \mathrm{C}$ for $10 \mathrm{~min}$ utes. The samples were size fractionated using SDSPAGE (precast $10 \%$ polyacrylamide gels; FMC, Philadelphia, Pennsylvania, USA) and transferred to Hybond-P (PVDF) membrane (Amersham Pharmacia Biotech). The anti-mEL antibody, used as the primary antibody, was diluted 1:2500, and the secondary HRP-conjugated goat anti-rabbit IgG (Jackson Labs, Bar Harbor, Maine, USA) was diluted 1:5000 from a 50\% glycerol stock. Detection was carried out by the ECL protocol (Amersham Pharmacia Biotech) according to the manufacturer's instructions. Chemiluminescence signals were detected on $\mathrm{x}$-ray films and quantified by densit-

\section{Figure 1}

Characterization of the inhibitory effects of the anti-mEL antibody and its specificity. (a) Conditioned media from HEK293 cells transfected with the $m E L c D N A$ were preincubated with increasing amounts of control IgG or anti-mEL IgG, and effects on triglyceride lipase and phospholipase activity were determined and compared with effects in untreated (PBS) media. The results are presented as the percentage of activity in medium preincubated with PBS alone and are the means \pm SD of triplicate determinations. (b) Conditioned media from HEK293 cells transfected with $\mathrm{mEL}, \mathrm{mHL}$, and $\mathrm{mLPL}$ cDNAs were preincubated with PBS, control IgG, or anti-mEL IgG, and the effects on triglyceride lipase activity were determined. The results are presented as the percentage of activity in medium preincubated with PBS alone and are the means \pm SD of triplicate determinations. (c) Homogenized liver lysates ( $15 \mu \mathrm{g}$ protein) from wildtype, human apoA-I transgenic, and $\mathrm{HL}^{-1-}$ mice and conditioned media from mEL-, $\mathrm{mHL}$-, and $\mathrm{mLPL}$-transfected cells were separated by SDS-PAGE and immunoblotted with an anti-mEL antibody. Both forms of $\mathrm{mEL}$ were detected in the liver lysates of all three mouse models and were of slightly smaller size than mELs in conditioned media. The additional band detected in the human apoA-I transgenic and $\mathrm{HL}^{-1-}$ mouse liver lysates was also seen on immunoblotting of these same liver lysates using a control antibody (data not shown). No bands were seen in the $\mathrm{mHL}$ or $\mathrm{mLPL}$ conditioned media.

ometry. A separate but identical membrane was immunoblotted using the control antibody.

In vivo inhibition of $m E L$. Female mice (C57BL/ 6 mice, $H L^{-/-}$mice [11], and human apoA-I transgenic mice) were obtained from Jackson Labs. Mice were maintained on a 12-hour light/dark cycle and fed a chow diet. Before each study, serum cholesterol levels were determined, and the mice were divided into two groups such that the mean plasma cholesterol levels in the groups were not different. Each group contained five or six mice.

To determine the effects of inhibition of EL in vivo, we calculated the amount of antibody that was sufficient to inhibit the majority of EL phospholipase activity in mouse plasma in vitro. One milligram of antimEL IgG was sufficient to inhibit 2,200 units of $\mathrm{mEL}$ activity, which approximates the amount of postheparin phospholipase activity in $H L^{-/-}$mice. Mice were injected intravenously through the tail vein with $1 \mathrm{mg}$ of either anti-mEL or control rabbit IgG. Blood was obtained from the retro-orbital plexus at various times before and after antibody injection. Mice were fasted 4 hours before bleeding, and blood was always drawn at the same time of day. Aliquots of plasma were stored at $-20^{\circ} \mathrm{C}$ until analysis of lipids. Plasma cholesterol, 
HDL-C, and triglyceride levels were measured enzymatically on a Cobas Fara II (Roche Diagnostic Systems Inc., Indianapolis, Indiana, USA) using SigmaAldrich (St. Louis, Missouri, USA) reagents. Non-HDL cholesterol levels were determined by subtracting HDL-C from the total cholesterol. Plasma samples ( $200 \mu \mathrm{l}$ pooled from each group) were analyzed by fast protein liquid chromatography (FPLC, Amersham Pharmacia Biotech) on two Superose 6 columns in series as described previously (1). The cholesterol concentrations in the FPLC fractions were determined using an enzymatic assay (Wako Pure Chemical Industries Ltd., Osaka, Japan).

HDL phospholipid turnover studies. We performed HDL phospholipid turnover studies using a protocol based on previously published studies $(12,13)$. Human total HDL $(d=1.063-1.21 \mathrm{~g} / \mathrm{ml})$ and lipoprotein-deficient plasma $(d=1.21 \mathrm{~g} / \mathrm{ml})$ were prepared by ultracentrifugation as previously described (14). HDL was labeled with $\left[{ }^{3} \mathrm{H}\right]$ methylcholine-dipalmitoyl phosphatidylcholine $\left(\left[{ }^{3} \mathrm{H}\right]\right.$ methylcholine-DPPC) (PerkinElmer Life Sciences, Boston, Massachusetts, USA) in a manner similar to that used to label HDL with cholesteryl ester (15). Briefly, HDL were dialyzed against PBS containing $0.01 \%$ EDTA. Fifty microcuries of $\left[{ }^{3} \mathrm{H}\right]$ methylcholine-DPPC was dried under nitrogen in a glass tube and resuspended in $50 \mu \mathrm{l}$ of ethanol. Two milligrams of HDL protein was mixed with approximately $100 \mathrm{mg}$ of lipoprotein-deficient plasma protein in a glass tube, and the $\left[{ }^{3} \mathrm{H}\right]$ methylcholine-DPPC was added dropwise with intermittent vortexing. The tube was purged with nitrogen, sealed, and placed in a shaking water bath at $37^{\circ} \mathrm{C}$ for 24 hours. After incubation, labeled HDL was reisolated at the original density, dialyzed against PBS, and filter sterilized before injection into mice.

Eight female apoA-I transgenic mice (6-8 weeks of age) were injected with $1 \mathrm{mg}$ of either anti-mEL $(n=4)$ or control $\operatorname{IgG}(n=4)$. One hour after injection, mice were injected through the tail vein with $\left[{ }^{3} \mathrm{H}\right]$ methylcholine-DPPC-labeled HDL (approximately $1 \times 10^{6}$ $\mathrm{dpm}$ in $100 \mu \mathrm{l}$ of PBS). A timer was started immediately after HDL injection, and blood samples were obtained from the retro-orbital plexus at $1,5,15$, and 30 minutes and at 1, 2, 4, and 6 hours. Six microliters of plasma taken at each time point were counted using a Beckman Coulter LS 6500 (Beckman, Fullerton, California, USA). The fractional catabolic rates of HDL phospholipid were calculated with the SAAM II program (SAAM Institute, Seattle, Washington, USA) by fitting a biexponential curve to the plasma counts normalized to the 1-minute time point.

Statistical analysis. Data are presented as means $\pm \mathrm{SD}$. Data analyses were performed using the Student's $t$ test. A value of $P<0.05$ was considered significant.

\section{Table 1}

\section{Results}

Like human EL (3), murine EL exhibits both triglyceride lipase and phospholipase activities. The ability of rabbit anti-mEL IgG to inhibit both the triglyceride lipase and phospholipase activities of mEL was examined (Figure 1a). Control rabbit IgG had no effect on the triglyceride lipase or phospholipase activities of mEL. In contrast, there was dose-dependent inhibition of both types of $\mathrm{mEL}$ lipase activities by anti-mEL IgG. Using $50 \mu \mathrm{g}$ per assay tube of anti-mEL IgG, 58\% of the triglyceride lipase and $100 \%$ of the phospholipase activities were inhibited. In another experiment, we examined whether the anti-mEL antibody can specifically inhibit $\mathrm{mEL}$ activity but not mHL and mLPL. As shown in Figure 1b, anti-mEL IgG did not inhibit the triglyceride lipase activity of $\mathrm{mHL}$ and $\mathrm{mLPL}$. In addition, the specificity of this antibody was examined by immunoblotting conditioned media containing $\mathrm{mEL}$, $\mathrm{mHL}$, and $\mathrm{mLPL}$. As shown in Figure 1c, the antibody detected mEL well but had no reactivity with mHL or mLPL. Finally, in order to confirm that the anti-mEL antibody had no crossreactivity with $\mathrm{mHL}$ in mouse liver, it was used for immunoblotting of mouse liver lysate; bands consistent with the two forms of $\mathrm{mEL}$ were seen, but no band consistent with $\mathrm{mHL}$ was seen (Figure 1c).

We examined the effects of an injection of the anti$\mathrm{mEL}$ antibody on plasma lipids in female wild-type mice. As indicated in Table 1, plasma levels of total cholesterol, HDL-C, and phospholipid were significantly increased after injection of anti-mEL IgG as compared with control IgG at both 24 hours and 48 hours after injection. Triglyceride and non-HDL cholesterol levels were not changed. The distribution of cholesterol among the lipoprotein classes was determined by FPLC separation of lipoproteins in pooled plasma samples (Figure 2a). This confirmed that HDL-C was substantially increased in the mice injected with anti-mEL IgG as compared with those injected with control IgG.

Although the anti-mEL antibody did not cross-react with murine HL on Western blotting and did not inhibit mHL activity in vitro, we performed an in vivo experiment in female $\mathrm{HL}^{-/-}$mice in order to exclude the possibility that the effects on HDL-C in wild-type mice could have been due to cross-inhibition of HL by the anti-mEL antibody. In $\mathrm{HL}^{-/-}$mice, plasma levels of

Plasma lipids in wild-type mice after IgG injection

\begin{tabular}{lcccccccc}
\hline & \multicolumn{4}{c}{ Control $(n=5)$} & \multicolumn{4}{c}{ Anti-mEL $(n=5)$} \\
mg/dl (SD) & TC & HDL-C & TG & PL & TC & HDL-C & TG & PL \\
Baseline & 75.0 & 60.5 & 53.8 & 131.0 & 77.8 & 64.3 & 56.6 & 130.3 \\
& $(8.2)$ & $(4.8)$ & $(3.2)$ & $(12.8)$ & $(10.1)$ & $(7.4)$ & $(5.0)$ & $(18.9)$ \\
\multirow{3}{*}{24 hours } & 92.2 & 62.0 & 85.3 & 145.8 & $111.3^{\mathrm{B}}$ & $80.3^{\mathrm{A}}$ & 74.1 & $185.7^{\mathrm{B}}$ \\
& $(8.6)$ & $(5.0)$ & $(11.7)$ & $(21.5)$ & $(6.4)$ & $(4.9)$ & $(3.1)$ & $(11.0)$ \\
48 hours & 95.1 & 70.7 & 80.2 & 172.0 & $125.5^{\mathrm{A}}$ & $92.8^{\mathrm{A}}$ & 80.3 & $205.0^{\mathrm{A}}$ \\
& $(10.4)$ & $(4.9)$ & $(12.1)$ & $(14.0)$ & $(5.0)$ & $(2.3)$ & $(4.2)$ & $(9.6)$ \\
& & & & & & & &
\end{tabular}

TC, total cholesterol; TG, triglyceride; PL, phospholipid. ${ }^{A} P<0.001$ as compared with control. ${ }^{\mathrm{B}} \mathrm{P}<0.05$ as compared with control. 


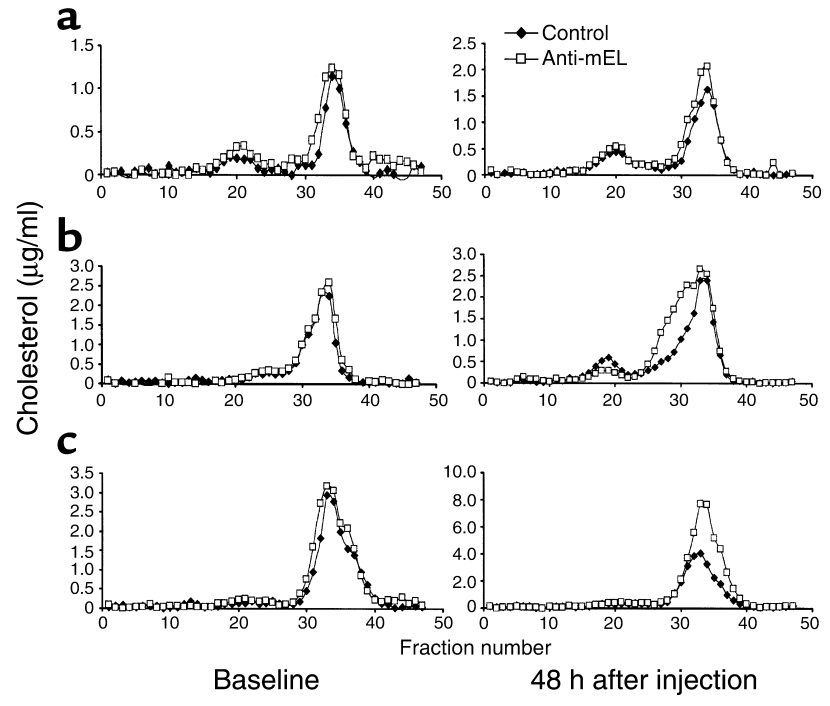

Figure 2

Plasma lipoprotein profiles at baseline and after antibody injection. Pooled plasma samples obtained at baseline and 48 hours after control or anti-mEL IgG injection were subjected to FPLC using Superose 6 columns, and the cholesterol concentration in each fraction was measured. (a) Wild-type mice. (b) $\mathrm{HL}^{-/-}$mice. (c) Human apoA-I transgenic mice.

HDL-C, total cholesterol, and phospholipid were substantially increased after anti-mEL antibody administration, with the peak at 48 hours after injection (Table 2). FPLC of pooled plasma samples (Figure $2 b$ ) confirmed that HDL-C was increased in the group injected with anti-mEL antibody. Furthermore, the size of the HDL particles after inhibition of EL in $\mathrm{HL}^{-/-}$mice was noticeably larger. A similar increase in phospholipids in large HDL particles was seen in mice injected with anti-mEL IgG (data not shown). In contrast to wild-type mice, triglyceride levels in $H L^{-/-}$mice were also increased at 48 hours and 72 hours after injection of anti-mEL antibody (Table 2). A second independent study was done in male $H L^{-/-}$mice, and comparable results were obtained (data not shown).

Human apoA-I transgenic mice have HDL that is more heterogeneous than mouse HDL and more similar to human HDL. Therefore, we performed another experiment of EL inhibition in human apoA-I transgenic mice. Plasma levels of total cholesterol, HDL-C, phospholipids, and apoA-I were significantly increased up to 72 hours after anti-mEL antibody administration, with the peak at 48 hours after injection (Table 3). FPLC of pooled plasma samples (Figure 2c) confirmed that HDL-C was markedly increased in the group injected with anti-mEL but not in the control group. Triglyceride and non-HDL cholesterol levels were not changed.

\section{Table 2}

We hypothesized that inhibition of EL raised HDL-C, phospholipid, and apoA-I levels by inhibiting the ELmediated hydrolysis of HDL phospholipids, thus slowing the turnover. Therefore, we directly investigated the effect of EL inhibition on the turnover of HDL phospholipid by intravenously injecting human apoA-I transgenic mice with anti-mEL or control IgG, followed by injection with HDL that was labeled with $\left[{ }^{3} \mathrm{H}\right]$ methylcholine-DPPC. The clearance of HDL phospholipid was significantly slower in mice injected with anti-mEL antibody than in mice injected with control antibody (Figure 3); the fractional catabolic rates were $0.42 \pm 0.03$ pools per hour and $0.53 \pm 0.02$ pools per hour, respectively $(P<0.0007)$. These results demonstrate that EL inhibition significantly retarded HDL phospholipid turnover in vivo.

\section{Discussion}

We used a rabbit polyclonal antibody inhibitory to $\mathrm{mEL}$ to demonstrate that inhibition of EL in mice results in substantial increases in HDL-C levels in vivo. These results were obtained in wild-type mice, $\mathrm{HL}^{-/-}$ mice, and human apoA-I transgenic mice. Plasma phospholipid levels were significantly increased as well by EL inhibition in each mouse model. In human apoA-I transgenic mice, apoA-I levels were also significantly increased by EL inhibition. In $H L^{-/-}$mice, but not in wild-type or human apoA-I transgenic mice, the size of the HDL increased after EL inhibition. The turnover of HDL phospholipids was significantly slowed by EL inhibition. Although EL has triglyceride lipase activity in vitro, acute inhibition of EL did not result in a change in plasma triglyceride levels except in $\mathrm{HL}^{-/-}$ mice. These studies provide the first in vivo data demonstrating that inhibition of EL influences HDL metabolism and support the concept that HDL is a physiological substrate for EL.

The use of antibody inhibition to elucidate the physiological roles of members of this lipase family in vivo is well established. Antibody inhibition of LPL in monkeys (8) and roosters (9) resulted in a rapid increase of triglyceride-rich large VLDL and a small decrease in HDL cholesterol. Inhibition of HL in rats $(4-6,16)$ and monkeys (7) resulted in a rapid increase in triglyceride-rich small

Plasma lipid in $\mathrm{HL}^{-/-}$mice after $\operatorname{lgG}$ injection

\begin{tabular}{lcccccccc}
\hline & \multicolumn{3}{c}{ Control $(n=6)$} & \multicolumn{4}{c}{ Anti-mEL $(\boldsymbol{n}=6)$} \\
mg/dl (SD) & TC & HDL-C & TG & PL & TC & HDL-C & TG & PL \\
Baseline & 156.0 & 113.4 & 62.4 & 291.8 & 168.0 & 113.6 & 55.0 & 293.8 \\
& $(22.4)$ & $(19.6)$ & $(16.1)$ & $(33.9)$ & $(33.8)$ & $(16.8)$ & $(13.2)$ & $(49.9)$ \\
\multirow{3}{*}{ 24 hours } & 166.6 & 112.0 & 91.2 & 340.6 & 210.8 & $133.4^{\mathrm{A}}$ & 101.0 & $451.4^{\mathrm{B}}$ \\
& $(24.8)$ & $(10.7)$ & $(6.4)$ & $(18.4)$ & $(31.0)$ & $(18.6)$ & $(14.4)$ & $(62.8)$ \\
48 hours & 167.8 & 105.6 & 62.8 & 321.8 & $242.2^{\mathrm{A}}$ & $159.6^{\mathrm{A}}$ & $102.0^{\mathrm{A}}$ & $467.6^{\mathrm{A}}$ \\
& $(25.9)$ & $(14.9)$ & $(15.9)$ & $(31.6)$ & $(35.2)$ & $(21.8)$ & $(18.8)$ & $(63.2)$ \\
72 hours & 158.0 & 105.6 & 57.0 & 291.0 & $219.6^{\mathrm{B}}$ & 143.4 & $82.2^{\mathrm{A}}$ & $410.6^{\mathrm{A}}$ \\
& $(28.6)$ & $(18.5)$ & $(18.9)$ & $(33.8)$ & $(28.3)$ & $(19.1)$ & $(12.6)$ & $(41.0)$
\end{tabular}

TC, total cholesterol; TG, triglyceride; PL, phospholipid. ${ }^{A} P<0.001$ as compared with control. в $P<0.05$ as compared with control. 
Table 3

Plasma lipids and apoA-I in apoA-I transgenic mice after IgG injection

\begin{tabular}{|c|c|c|c|c|c|c|c|c|c|c|}
\hline \multirow[b]{2}{*}{$\mathrm{mg} / \mathrm{dl}(\mathrm{SD})$} & \multicolumn{5}{|c|}{ Control $(n=6)$} & \multicolumn{5}{|c|}{ Anti-mEL $(n=6)$} \\
\hline & TC & HDL-C & TG & PL & ApoA-I & $\mathrm{TC}$ & HDL-C & TG & PL & ApoA-I \\
\hline Baseline & $\begin{array}{c}211.3 \\
(24.9)\end{array}$ & $\begin{array}{c}171.0 \\
(19.4)\end{array}$ & $\begin{array}{l}60.8 \\
(7.9)\end{array}$ & $\begin{array}{l}362.8 \\
(52.8)\end{array}$ & $\begin{array}{l}345.5 \\
(55.4)\end{array}$ & $\begin{array}{l}218.0 \\
(20.7)\end{array}$ & $\begin{array}{c}175.0 \\
(17.4)\end{array}$ & $\begin{array}{c}67.8 \\
(17.7)\end{array}$ & $\begin{array}{l}366.8 \\
(33.4)\end{array}$ & $\begin{array}{c}347.5 \\
(42.6)\end{array}$ \\
\hline 24 hours & $\begin{array}{c}257.7 \\
(25.8)\end{array}$ & $\begin{array}{c}196.0 \\
(23.3)\end{array}$ & $\begin{array}{l}105.5 \\
(20.1)\end{array}$ & $\begin{array}{l}443.5 \\
(40.0)\end{array}$ & $\begin{array}{l}400.0 \\
(25.9)\end{array}$ & $\begin{array}{l}297.2^{B} \\
(31.0)\end{array}$ & $\begin{array}{c}234.5^{\mathrm{A}} \\
(17.9)\end{array}$ & $\begin{array}{l}172.8 \\
(72.4)\end{array}$ & $\begin{array}{l}569.8^{\mathrm{B}} \\
(66.4)\end{array}$ & $\begin{array}{l}546.5^{\mathrm{A}} \\
(40.6)\end{array}$ \\
\hline 48 hours & $\begin{array}{l}255.3 \\
(23.7)\end{array}$ & $\begin{array}{r}196.5 \\
(13.5)\end{array}$ & $\begin{array}{l}84.3 \\
(9.8)\end{array}$ & $\begin{array}{l}418.8 \\
(32.6)\end{array}$ & $\begin{array}{l}369.0 \\
(48.2)\end{array}$ & $\begin{array}{c}318.2^{\mathrm{A}} \\
(9.3)\end{array}$ & $\begin{array}{c}257.0^{\mathrm{A}} \\
(8.8)\end{array}$ & $\begin{array}{c}84.3 \\
(12.9)\end{array}$ & $\begin{array}{l}538.7^{\mathrm{A}} \\
(14.0)\end{array}$ & $\begin{array}{l}511.5^{\mathrm{A}} \\
(50.4)\end{array}$ \\
\hline 72 hours & $\begin{array}{l}188.2 \\
(8.0)\end{array}$ & $\begin{array}{l}152.5 \\
(13.4)\end{array}$ & $\begin{array}{c}60.2 \\
(17.5)\end{array}$ & $\begin{array}{l}310.7 \\
(31.6)\end{array}$ & $\begin{array}{l}330.0 \\
(26.5)\end{array}$ & $\begin{array}{l}239.5^{\mathrm{A}} \\
(17.6)\end{array}$ & $\begin{array}{l}235.2^{\mathrm{A}} \\
(34.4)\end{array}$ & $\begin{array}{c}63.3 \\
(17.3)\end{array}$ & $\begin{array}{c}383.5^{\mathrm{B}} \\
(51.8)\end{array}$ & $\begin{array}{c}393.5^{\mathrm{B}} \\
(51.1)\end{array}$ \\
\hline
\end{tabular}

TC, total cholesterol; TG, triglyceride; PL, phospholipid. ${ }^{A} P<0.001$ as compared with control. ${ }^{\mathrm{B} P}<0.05$ as compared with control.

VLDL and IDL with a modest increase in HDL. These antibody inhibition studies provided major insights into the physiological roles of LPL and HL.

Although we showed that the anti-mEL antibody does not cross-inhibit $\mathrm{mHL}$ and $\mathrm{mLPL}$ activity in vitro, we performed two independent studies in $H L^{-/-}$mice in order to exclude the possibility that these effects on $\mathrm{HDL}$ could be due to HL inhibition. Even in $\mathrm{HL}^{-/-}$mice, HDL-C levels were significantly increased by injection of anti-mEL antibody, confirming that this result is not the result of inhibition of HL. Although to our knowledge HL antibody inhibition studies have not been reported in mice, studies in rats $(4-6,16)$ indicated only moderate increases in HDL-C with acute HL inhibition. Our studies suggest that reduction in EL activity may have a more substantial effect on HDL than reduction in HL activity. Further studies involving side-by-side comparisons and simultaneous inhibition of both enzymes will be required in order to gain further insight into the relative roles of $\mathrm{HL}$ and $\mathrm{EL}$ in HDL metabolism.

HDL particle size was increased by EL inhibition in $\mathrm{HL}^{-/-}$mice, suggesting an interaction between $\mathrm{HL}$ and EL in remodeling HDL and influencing HDL particle size. The influence of EL inhibition on HDL particle size appears to depend on the metabolic milieu, as there were no obvious effects on HDL particle size as assessed by FPLC fractionation of lipoproteins in wild-type or human apoA-I transgenic mice. $H L^{-/-}$mice have larger HDL particles with increased phospholipid content (11), and these HDL particles may be especially good substrates for EL. It is interesting to note that $\mathrm{HL}^{-/-}$ mice were also the only group that had an increase in triglyceride levels with EL inhibition, suggesting that the effects of EL on triglyceride-rich lipoprotein metabolism may also be magnified in the absence of HL.

Our studies also demonstrated that mice injected with anti-mEL antibody had a significantly slower HDL phospholipid catabolic rate than control antibody-injected mice. This supports the concept that inhibition of EL phospholipase activity reduces the rate of HDL phospholipid turnover. Detailed studies of HDL composition after anti-mEL antibody injection compared to preinjection and to control antibody injection demonstrated that the relative composition of the HDL particles was not significantly changed by inhibition of EL (data not shown). This suggests that although the initial effect of EL inhibition may be inhibition of HDL phospholipid hydrolysis, the net effect is to influence the overall number of HDL particles. The change in the HDL phospholipid fractional catabolic rate during the first 5 hours after anti-mEL antibody injection was $21 \%$, yet plasma levels of HDL-C and phospholipids were both increased by $47 \%$ at 48 hours after injection. These increases cannot be accounted for solely by reduced catabolism and might be due in part to increased "production" of HDL cholesterol and phospholipids, perhaps by transfer from other lipoproteins or from tissues. It is possible that the increases in HDL due to delayed catabolism result secondarily in a greater efflux of cholesterol and phospholipids from peripheral tissues. Future studies will focus on whether inhibition of EL promotes lipid efflux.

Our current working model is that EL, like LPL and HL, is secreted and then binds to heparan sulfate proteoglycans on the endothelial surface. There, it interacts with HDL, hydrolyzing HDL phospholipids and generating free fatty acids at the local tissue site. The end result of this process, analogous to the interaction of LPL and triglyceride-rich lipoproteins, is the generation of a

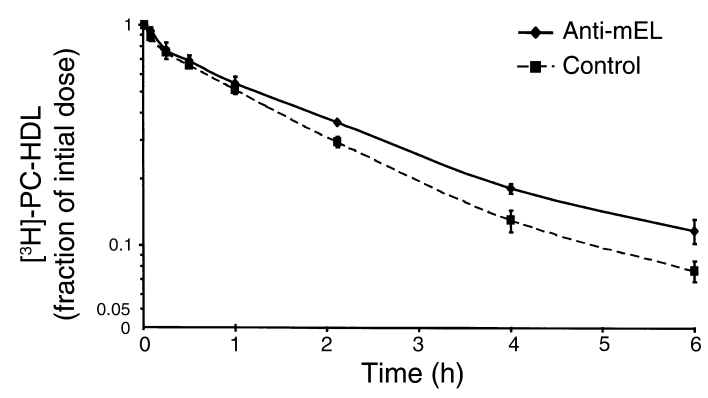

\section{Figure 3}

The clearance of HDL phospholipid from plasma in human apoA-I transgenic mice injected with either anti-mEL or control IgG. Mice ( $n=4$ mice per group) were injected with either anti-mEL (diamonds) or control IgG (squares). One hour later mice were injected through the tail vein with $\left[{ }^{3} \mathrm{H}\right]$ methylcholine-DPPC-labeled $\mathrm{HDL}\left(\left[{ }^{3} \mathrm{H}\right]-\mathrm{PC}-\right.$ $\mathrm{HDL}$ ), and blood samples were obtained over a 6-hour period. Six microliters of plasma taken at each time point were counted, and data are expressed as fractions of the counts at 1 minute. 
"remnant" HDL particle depleted in phospholipids that is preferentially catabolized. According to this model, inhibition of EL reduces HDL phospholipid hydrolysis, slows HDL turnover, and increases HDL phospholipid, cholesterol, and apoA-I levels. In summary, the studies presented here demonstrate for the first time that reduction of EL activity in vivo has significant effects on HDL-C levels and suggest that EL may be an important enzyme in the regulation of HDL metabolism.

\section{Acknowledgments}

We thank Anna Lillethun, Linda Morrell, Nadine Blanchard, Anthony Secreto, and Dawn Marchadier for technical assistance. This work was supported by a Burroughs Wellcome Foundation Clinical Scientist Award in Translational Research and NIH grant HL55323 from the National Heart Lung and Blood Institute to D.J. Rader, and by NIH grant M01-RR00040 from the NCRR supporting the University of Pennsylvania General Clinical Research Center. D.J. Rader is an Established Investigator of the American Heart Association.

1. Jaye, M., et al. 1999. A novel endothelial-derived lipase that modulates HDL metabolism. Nat. Genet. 21:424-428.

2. Hirata, K., et al. 1999. Cloning of a unique lipase from endothelial cells extends the lipase gene family. J. Biol. Chem. 274:14170-14175.

3. McCoy, M.G., et al. 2002. Characterization of the lipolytic activity of endothelial lipase. J. Lipid Res. 43:921-929.
4. Kuusi, T., Kinnunen, P.K., and Nikkila, E.A. 1979. Hepatic endothelial lipase antiserum influences rat plasma low and high density lipoproteins in vivo. FEBS Lett. 104:384-388.

5. Grosser, J., Schrecker, O., and Greten, H. 1981. Function of hepatic triglyceride lipase in lipoprotein metabolism. J. Lipid Res. 22:437-442.

6. Murase, T., and Itakura, H. 1981. Accumulation of intermediate density lipoprotein in plasma after intravenous administration of hepatic triglyceride lipase antibody in rats. Atherosclerosis. 39:293-300.

7. Goldberg, I.J., et al. 1982. Lipoprotein metabolism during acute inhibition of hepatic triglyceride lipase in the cynomolgus monkey. J. Clin. Invest. 70:1184-1192.

8. Goldberg, I.J., Le, N.A., Ginsberg, H.N., Krauss, R.M., and Lindgren, F.T. 1988. Lipoprotein metabolism during acute inhibition of lipoprotein lipase in the cynomolgus monkey. J. Clin. Invest. 81:561-568.

9. Sato, K., Akiba, Y., Chida, Y., and Takahashi, K. 1999. Lipoprotein hydrolysis and fat accumulation in chicken adipose tissues are reduced by chronic administration of lipoprotein lipase monoclonal antibodies. Poult. Sci. 78:1286-1291.

10. Page, M., and Thorpe, R. 1998. IgG purification. Methods Mol. Biol. 80:95-111.

11. Homanics, G.E., et al. 1995. Mild dyslipidemia in mice following targeted inactivation of the hepatic lipase gene. J. Biol. Chem. 270:2974-2980.

12. Pownall, H.J., Hickson-Bick, D., and Massey, J.B. 1991. Effects of hydrophobicity on turnover of plasma high density lipoproteins labeled with phosphatidylcholine ethers in the rat. J. Lipid Res. 32:793-800.

13. Foger, B., et al. 1997. Plasma phospholipid transfer protein. Adenovirusmediated overexpression in mice leads to decreased plasma high density lipoprotein (HDL) and enhanced hepatic uptake of phospholipids and cholesteryl esters from HDL. J. Biol. Chem. 272:27393-27400.

14. Hatch, F.T. 1968. Practical methods for plasma lipoprotein analysis. $A d v$. Lipid Res. 6:1-68.

15. Terpstra, A.H., Nicolosi, R.J., and Herbert, P.N. 1989. In vitro incorporation of radiolabeled cholesteryl esters into high and low density lipoproteins. J. Lipid Res. 30:1663-1671.

16. Jansen, H. 1985. Inhibition of liver lipase in vivo leads to induction of de novo cholesterol synthesis in rat liver. Biochem. Biophys. Res. Commun. 131:574-578. 